\title{
The Use of Lane-Centering to Ensure the Visible Light Communication Connectivity for a Platoon of Autonomous Vehicles
}

\author{
Mohammad Y. Abualhoul ${ }^{\dagger}$, Edgar Talavera Munoz ${ }^{\ddagger}$, and Fawzi Nashashibi ${ }^{\dagger}$ \\ RITS Team, French Institute for Research in Computer Science and Automation (INRIA-Paris) ${ }^{\dagger}$ \\ Escuela Técnica Superior de Ingeniería de Sistemas, Universidad Politécnica de Madrid \\ \{mohammad.abu_alhoul, fawzi.nashashibi\}@inria.fr, e.talavera@upm.es
}

\begin{abstract}
The new emerging Visible Light Communication (VLC) technology has been subjected to intensive investigation, evaluation, and lately, deployed in the context of convoy-based applications for Intelligent Transportations Systems (ITS). The technology limitations were defined and supported by different solution proposals to enhance the crucial alignment and mobility limitations. In this paper, we propose to incorporate VLC technology and the Lane-Centering (LC) technique to ensure the VLC-connectivity by keeping the autonomous vehicle aligned to the lane center using vision-based lane detection in a convoy-based formation. As soon as the road lanes are detectable, the evaluated system showed stable behavior independently of the inter-vehicle distances and without the need to exchange information between vehicles. Such a combination can ensure the optical communication connectivity with a lateral error of less than $30 \mathrm{~cm}$. The evaluation of the proposed system is verified using VLC prototype and an empirical result of an LC active application over $60 \mathrm{~km}$ on the Madrid M40 highway.
\end{abstract}

Keywords: VLC, Lane-Centering, Platoon, ITS, Road safety applications.

\section{INTRODUCTION}

Nowadays, Intelligent Transportation Systems (ITS) promise to provide safer and more secure autonomous vehicular mobility by combining and utilizing vehicular sensors, control systems, different sorts of vehicular communication methods, and even specific driving formations. Moreover, by enabling the Cooperative ITS (C-ITS) mode, the cooperation between autonomous vehicles can provide the necessary remote and local data to perceive the vehicle's surrounding environment and raise the independent vehicles awareness. The proper information exchange between vehicles in convoybased applications can further improve both road traffic safety and string stability, especially when deploying fully autonomous applications [1], [2].

Due to the vast deployment of C-ITS services for vehicular applications, well-known wireless technologies such as the Dedicated Short-Range (DSR) communications for ITS [3], [4] are extensively used. These standardized vehicular communication technologies, including the widely deployed IEEE $802.11 \mathrm{p}$, are pushed lately toward an unstable performance for wireless networks data access, with a remarkable increase in both latency and channel congestion levels. This instability introduces more usage constraints for hard-safety ITS applications, for instance, the convoy-based applications [2], [5].

New communication solutions using an independent medium are being proposed for ITS applications, such as
VLC, which was recently proved to be a suitable supportive solution for radio-based communication in vehicular environments. In addition to the many advantages of deploying optical-based communication solutions, VLC can realize both low-cost implementation and dual functionality (communication signaling and lightning) [2], [6], [7]. However, the ambient noise (signal interference and optical noise) and the Field-of-View (FOV) (mobility issues) limitations are two main challenges of any VLC deployment for ITS applications. Various solutions have been proposed to deal with such constraints [8], [9]. Nevertheless, the Field-ofView (FOV) limitation, which is expressed as the maximum angular size of the Photo-diode (PD), remains as the primary mobility challenge when VLC-based platoon members face slight changes in driving course, or lane changing.

On the other hand, the lane detection technique has always been considered as a crucial cost-efficient and reliable enabler for many driving-assistance autonomous systems [10]. The concept of Lane-Centering (LC) is simplistic and depends on performing real-time processing of the lane detection data and restoring the autonomous vehicle's position by locating itself referring to the side lanes (no cooperation is required). Such a technique can be beneficial in tunnels scenarios or when the alignment of convoy-based vehicles is essential.

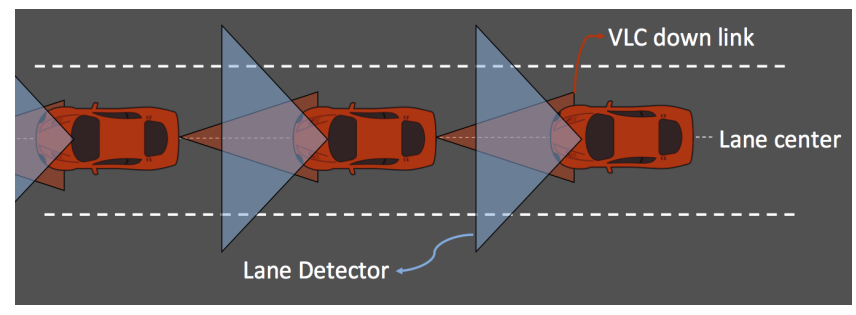

Fig. 1. Lane Detection and VLC down-link for platooning application.

In this paper, we propose the use of the LC technique to enhance the VLC inter-connectivity between the members of a platoon of autonomous vehicles, as depicted in Fig. 1. By combining both solutions (VLC and LC), the dependency on exchanging vehicular status and GPS positions will not be essential to relocate platoon members and ensure direct Lineof-Sight (LoS). Therefore, the main focus of our proposal is on preserving the alignment between platoon members with the minimum possible error and without requiring any 
information exchange between convoy members. Such a deployment is expected to allow the autonomous platoon members to return to the aligned status even during the VLC disturbance, which is likely to occur. In this paper, we make the following contributions:

- Suggesting a new architecture to enhance the platoon performance by utilizing both LC and VLC.

- Defining the limitations and performance of both technologies.

- Evaluating the overall system performance.

The remainder of this paper is organized as follows. Section II presents related work and a review of the literature. The over-all system proposal for platooning application enhancing VLC connectivity using LC is further explained in Section III. We report on the system's performance and our findings in Section IV. Finally, the conclusion and future work are given in Section V.

\section{RELATED WORK}

The convoy-based autonomous vehicles formation concept has been widely investigated and proposed to improve traffic fluidity by increasing road capacity while reducing fossil fuel consumption and $\mathrm{CO}_{2}$ levels. The leading PATH project in California [11] and PRAXITELE project in France [12] were the pioneering projects demonstrating the platoon application. Other projects such as Auto21 CDS [13] are dedicated to advancing smooth merging/leaving for a platooning-enabled system. Also, the SARTRE project [14] has successfully demonstrated the fully autonomous platoon.

At the vehicular control level, the most straightforward requirement to form a platoon is usually the longitudinal control, as described in [15], [16]. Simple approaches such as Adaptive Cruise Control (ACC) with a constant time headway policy has been a universal solution to ensure a safe inter-distance between platoon members [16].

Both conceptual and experimental studies on the usecase of VLC for outdoor applications have been conducted and presented for convoy-based ITS applications [17-19]. The studies and system evaluation have tackled the main technology limitations and use-cases showing that VLC can satisfy the stringent reachability requirements for InterVehicle Communication (IVC) in dense vehicle traffic conditions [20]. We at INRIA have also implemented our VLC prototype to evaluate and test the performance of VLC technology for platoon applications and examining real outdoor conditions [21].

Our previously conducted study [21] dedicated to evaluating the deployment of Laser-Range-Finder (LRF) and VLC for the same platoon application. The evaluation and system implementation were intended to provide a reliable backup system to switch the autonomous driving modes between ACC and Cooperative-ACC (C-ACC) to even making an emergency stop when failure scenario is detected.

Moreover, laser detection use-cases and deployment to improve vehicle awareness using different approaches, including the use of laser detection to implement obstacle detection applications, remains costly solution and requires a high computational process compared to Camera-based vision detection [22].

By using vision-detection, lane detection approach can offer either alternative or supportive solution to the laserbased-sensor systems compared with the previous proposal that uses Laser Range Finder (LRF) alongside the VLC in [21]. Further challenges to deploying vision-based lane detection for autonomous driving have also been discussed in the DARPA project [23].

Further contributions such as Wang in [10] propose the use of a B-Snake algorithm to improve lane detection models. Others, such as Hafner in [24] focus on the limitations of lane detection deployment for ITS and provide interesting mathematical solutions to overcome some computational and decision barriers. Lee has also presented a unique lane centering and control algorithm based on future predictions for autonomous driving maneuvers [25].

\section{Platoon CONTROL USING Both VLC AND LANE CENTERING}

In this section, we first present the classic kinematic longitudinal and lateral control model that governs the motion of an autonomous vehicle, and which was used to drive platoons in our previous studies [6], [21]. Afterward, we present the proposed LC control mechanism to apply longitudinalcorrection controller based on vision-based lane detection.

\section{A. Communication-based Platoon Control Model}

For simplicity, the deployed vehicle kinematics model was an approximation of the Ackerman steering mechanism (bicycle model). The bicycle model is defined by three main parameters, $\delta, l$, and $\phi$, which respectively represents the steering angle, wheelbase, and the orientation of the vehicle. Therefore, the kinematic model which governs the platoon members vehicle's motion was detailed in [26] using a constant steering angle $\delta$, with a circular vehicle's trajectory and a radius $R$ such as:

$$
\left\{\begin{array}{l}
l=R \cdot \tan (\delta) \\
\phi=\operatorname{atan}\left(\frac{\Delta y}{\Delta x}\right)-\theta \\
\Delta x=X_{L}-X_{F} \\
\Delta y=Y_{L}-Y_{F}
\end{array} .\right.
$$

where the steering angle $\delta$ is given by:

$$
\delta=\operatorname{atan}\left(\frac{2 \cdot l \cdot \sin (\phi)}{\sqrt{\Delta x^{2}+\Delta y^{2}}}\right) .
$$

Figure 2 depicts the general horizontal plan of two vehicles forming a platoon. The longitudinal and lateral distances $\Delta x$ and $\Delta y$ represent the projection of the inter-distance $d$. To estimate the value $d$, the controller must be aware and updated about $(\Delta x, \Delta y, \delta, \phi)$ in real-time, which will, therefore, allow proper longitudinal and lateral control corrections with some margin of error. The GPS location, vehicle orientation, and vehicle's relative speed are passed over one-hop to every preceding member in platoon chain (from preceding to follower vehicle). 


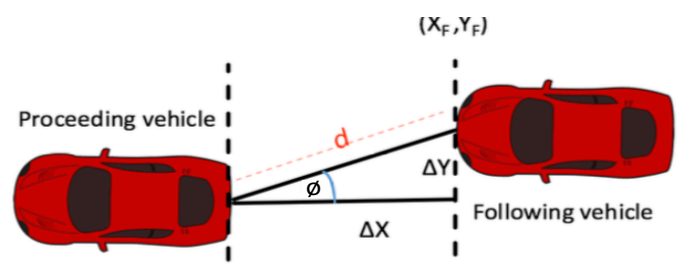

Fig. 2. Plan view of two vehicles platoon Leader (Proceeding) and Follower, which have the longitudinal $(\Delta x)$ and lateral $(\Delta y)$ displacements, inter-vehicle distance $(d)$.

Such requirements make it essential to have continuous and reliable communication between platoon members, and any disconnection or even data exchange latency can result in hazardous situations with potentially dangerous driving consequences.

\section{B. Lane Centering Model}

The LC concept can be expressed as a lateral control adjustment forcing the autonomous vehicle to center its position by continuously referring to the side lanes, and without the need for any further cooperative information.

Such lateral correction can be highly useful for convoybased applications. For platooning formations, ensuring members' centering can even remarkably improve the overall system string stability [27]. Furthermore, it becomes even more interesting when an optical-based communication system (VLC) is used to exchange information between platoon members. This approach allows every individual vehicle in convoy formation to locally change its behavior (orientation) to ensure a fixed position all along the driving path, even when communication is interrupted.

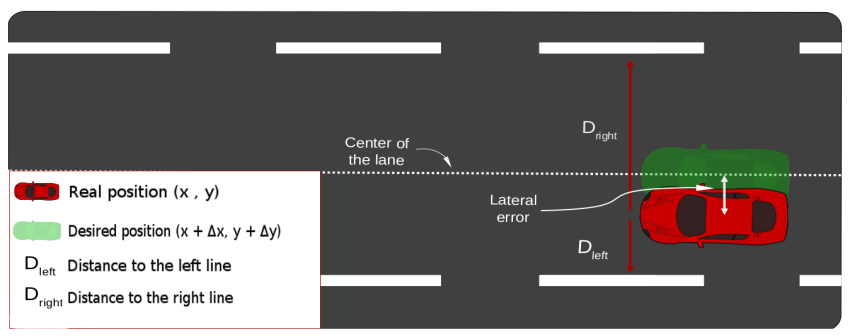

Fig. 3. System overview for Lane-Centering mechanism depicting the lateral error left and right values $\left(D_{\text {left }}, D_{\text {right }}\right)$ obtained by the artificial vision system.

Figure 3 illustrates the LC over-all detection mechanism and the applied lateral correction to compensate for the misalignment and reposition the desired vehicle (red vehicle) onto its desired location (lane center). The location error of the red vehicle represents the current position in UTM (Universal Transverse Mercator) coordinates $x$ and $y$; where both $D_{\text {left }}$ and $D_{\text {right }}$ represent the distance from the vehicle center point to the edges of the lanes.

By estimating the values of $D_{\text {left }}$ and $D_{\text {right }}$, and comparing them with the local vehicle position, the vehicle will have an initial awareness of the lateral error $\varepsilon$ and perform corrections based on orientation calculations to reach the desired position.

Estimating the lateral error $(\varepsilon)$ as defined by Equation 3 is the first step in executing correction, where $C_{\text {position }}$, represents the reference point (lane-center) obtained directly from the artificial vision sensor (MOBIL-EYE Camera in our validation [28]).

$$
\varepsilon= \begin{cases}\mathrm{D}_{\text {right }}-\mathrm{C}_{\text {position }}, & \text { if } \mathrm{C}_{\text {position }} \leq 0 \\ \mathrm{D}_{\text {left }}-\mathrm{C}_{\text {position }}, & \text { otherwise }\end{cases}
$$

To ensure proper autonomous driving orientation to position the vehicle on the lane-center; we use Equation 4 to calculate the exact displacement projection using the vehicle local GPS coordinations $(x, y)$, where $\delta x$ and $\delta y$ represent the error in both $x$ and $y$ coordinates.

$$
\begin{aligned}
& \delta x=\cos (\theta) * \varepsilon * \sigma_{\text {north }} \\
& \delta y=\sin (\theta) * \varepsilon * \sigma_{\text {east }}
\end{aligned}
$$

where the calculation of $\theta$ depends on $(\varepsilon)$ direction and the azimuth angle $(\dot{\alpha})$ as follows:

For $(\varepsilon<0)$ :

$$
\theta= \begin{cases}\dot{\alpha}, & 0^{\circ} \leq \dot{\alpha}<90^{\circ} \\ \dot{\alpha}-90, & 90^{\circ} \leq \dot{\alpha}<180^{\circ} \\ \dot{\alpha}-180, & 180^{\circ} \leq \dot{\alpha}<270^{\circ} \\ \dot{\alpha}-270, & \text { otherwise }\end{cases}
$$

For $(\varepsilon \geq 0)$ :

$$
\theta=\left\{\begin{array}{l}
90-\dot{\alpha}, \quad 0^{\circ} \leq \dot{\alpha}<90^{\circ} \\
180-\dot{\alpha}, \quad 90^{\circ} \leq \dot{\alpha}<180^{\circ} \\
270-\dot{\alpha}, \quad 180^{\circ} \leq \dot{\alpha}<270^{\circ} \\
360-\dot{\alpha}, \quad \text { otherwise }
\end{array}\right.
$$

As orientation adjustments are required to apply the right correction (front vehicle direction), both the azimuth angle $(\dot{\alpha})$ and the correction direction $(\sigma)$ are calculated as follows:

$$
\begin{aligned}
\sigma_{\text {north }} & = \begin{cases}-1, & \text { if } 270>\dot{\alpha} \geq 90 \\
+1, & \text { otherwise }\end{cases} \\
\sigma_{\text {east }} & = \begin{cases}-1, & \text { if } \quad \dot{\alpha} \leq 180 \\
+1, & \text { otherwise }\end{cases}
\end{aligned}
$$

The azimuth angle $(\dot{\alpha})$ is a crucial element to calculate all necessary lateral control corrections. An accurate estimation of $\dot{\alpha}$ can dramatically improve overall system performance. Therefore, a higher resolution ( 4-point method) shown in Fig. 4 is applied to stabilize the estimated values further and avoid any GPS errors compared to the conventional 2-point method.

Instead of using only 2 GPS points, we use 4 points to successfully calculate 3 full consecutive azimuth angle updates and apply a mean to the result after distributing empirically optimized weights using the following formula. 


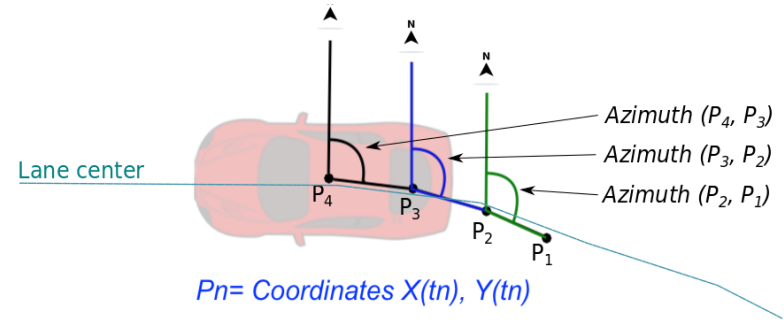

Fig. 4. The 4-point Azimuth angle calculation.

$$
\dot{\alpha}_{\text {final }}=\sum_{i=1}^{3} \frac{\dot{\alpha}_{i} * W_{i}}{3} \quad \mid \quad W_{i}=[0.5 ; 0.3 ; 0.2]
$$

TABLE I

LANE CENTERING CAMERA (MOBIL-EYE) PARAMETERS

\begin{tabular}{ll}
\hline Artificial Vision Camera Parameter & Value \\
\hline Model & Mobileye 5 Series \\
Transmission data Rate & $10 \mathrm{~Hz}$ \\
Focus range & $4 \mathrm{~m}$ to infinity \\
Angle of view & 35 \\
Pixel size & $6.0 \mu \mathrm{m} \times 6.0 \mu \mathrm{m}$
\end{tabular}

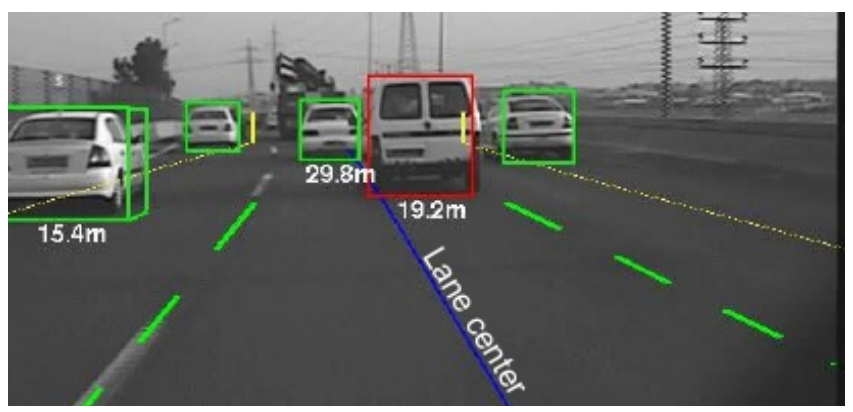

Fig. 5. A sample of the real-time artificial vision camera with detected objects for autonomous driving using Mobileye.

The platooning lane detection evaluations have used Mobileye [28] with the detailed parameters in Table I. The Mobileye refresh rate was tuned to limit vision to $\sim 4 \mathrm{~m}$ to avoid any conflict with other platoon members or road users. A further sample image of the real-time detection is depicted in Fig. 5.

\section{Visible Light Communication Model}

The definition of the VLC channel characteristics is strongly affected by several physical parameters such as the LED Lambertian emission pattern and both transmitter and receiver orientation. From Fig. 6, the channel path-loss can be presented as:

$$
P_{\text {loss }}=\left\{\begin{array}{cc}
\frac{(m+1) A_{p h}}{2 \pi d^{n}} \cos ^{m}(\varphi) \cos (\psi), & 0<\psi, \varphi<\theta_{c} \\
0 & \text { elsewhere }
\end{array},\right.
$$

where $\theta_{c}$ is the maximum incidence and irradiance angles $\max (\psi, \varphi) . A_{p h}$ is the physical area of the PD and $m=$ $-\frac{\ln 2}{\ln (\cos \hat{\phi})}$ is the Lambertian emission order, which is a key parameter specifying the directivity of the transmitter [6].

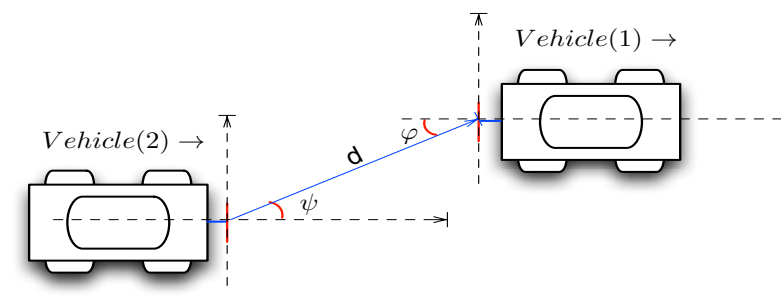

Fig. 6. Two-vehicle VLC-based platoon, with an inter-vehicle distance $d$ showing the incidence and irradiance angles $(\psi, \varphi)$ used to calculate the path-loss.

As mentioned earlier, we conducted an experimental system evaluation using a VLC prototype for platoon applications in [18]. The evaluation confirmed the inverse square proportional relationship between inter-vehicle distance $d$ and the received power for a link range of up to 30 meters in outdoor conditions.

The path-loss exponent $n$ found to be 2 for various weather situations and also sensitive to any misalignment which is the exact reason to deploy assisting control application such as the LC in this work. For more configuration details on both the physical and packet level see Table II.

TABLE II

VLC CONNECTIVITY AND RANGE EXPERIMENTAL PARAMETERS

\begin{tabular}{ll}
\hline VLC Platform Parameter & Value \\
\hline Transmission data Rate & $9.5 \mathrm{kbps}$ \\
Link maximum range & $30 \mathrm{~meter}$ \\
LED wavelength & $635 \mathrm{~nm}$ (RED) \\
Line coding \& Modulation & Manchester, OOK \\
LED power dissipation & 3 Watt \\
Number of LED array & 6 \\
LED lens viewing angle & $35^{\circ}$ \\
PD active area & $100 \mathrm{~mm}^{2}$ \\
Transmitter \& Receiver heights & $85 \mathrm{~cm}$
\end{tabular}

\section{System EVALUATION}

The performance of the proposed platoon system deploying both VLC and LC is evaluated by independently defining the following:

- The $90 \%$ of the received power defining the lateral area at the receiver side over different inter-vehicle distances, which represents the reliable performance of the VLC link with better immunity against ambient noise.

- The lateral control error before and after using the LC application, and how accurately the system can reposition the platoon members on the lane center.

To validate and test the proposed LC system limitations, two vehicles (Mitsubishi IMIEV) where equipped with an LC (using Mobileye camera). For initial positioning, one vehicle was located at the far left edge of the lane, while the second was located on the far right side of the same lane, as depicted Fig. 6. 
The experimentally acquired and processed data are of two vehicles driven in a platoon mode. Figure 7 represents sample results of the second vehicle's deviation variation from lanecenter. The deviation variation over the time interval $(0$ to 400 seconds) represents the case when the LC system is inactive; this can explain the remarkable misalignment which can reach up to 1-meter lateral error over 10 meters of intervehicle distance, which is outside the VLC lateral coverage limitations for reliable connection as shown in Fig. 8.

On the other hand, over the time interval (400 to 100 seconds), the deviation is reduced to less than $20 \mathrm{~cm}$ on average just after the LC is activated.

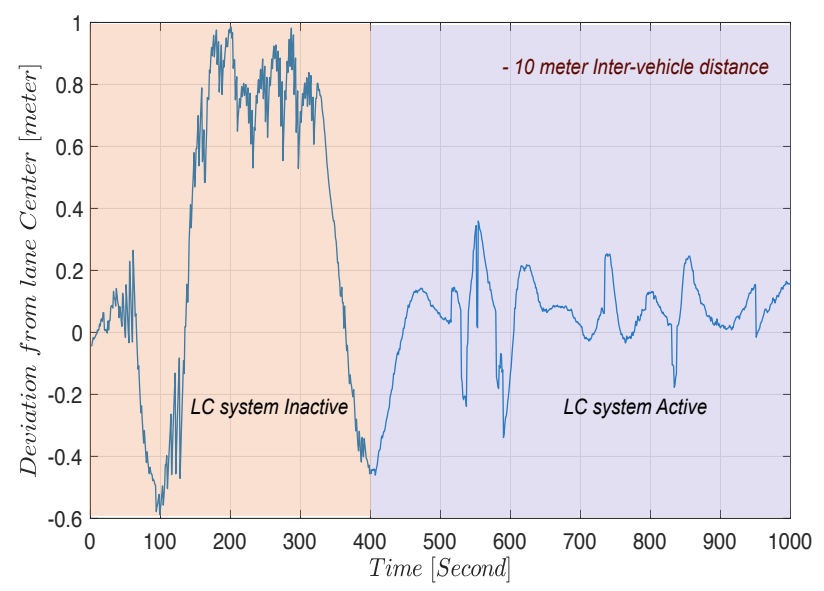

Fig. 7. Data Sample of a platoon member deviation from lane-center before and after activating the LC application.

To further understand how the activation of the LC system can ensure the VLC connectivity, we provide empirical results of the VLC link range tests using an actual VLC prototype. Figure 8 map the VLC link optical beam spot (spot diameter represent the lateral covering area) over different inter-vehicle distances. In Fig. 8, and for a vehicle distance of 10 meters, the misalignment is limited to $90 \mathrm{~cm}$ from lane center to guarantee a detectable signal level with an adequately received signal over noise ratio (SNR).

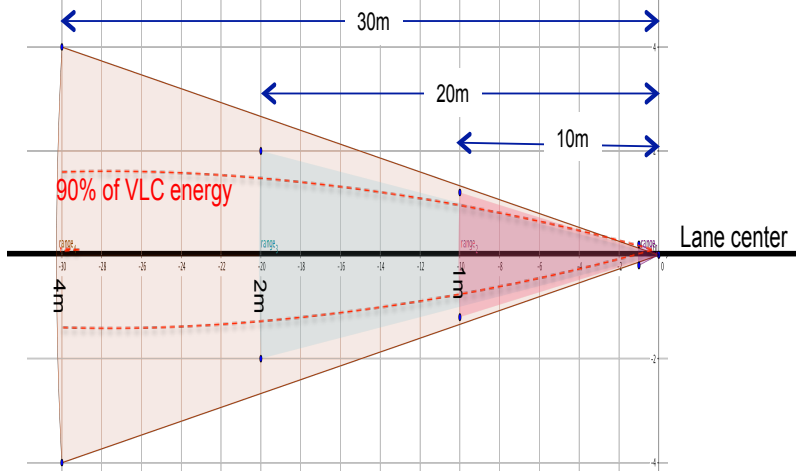

Fig. 8. The three main zones where the VLC receiver was placed to evaluate the received signal level. The experiment was conducted with respect to variations in direct distance changes and out-door conditions.
Other scenarios were experimentally validated as depicted in Fig. 9, which represents the same second platoon member's deviation data but for an autonomous driving path of $60 \mathrm{~km}$ as depicted in Fig. 10. The obtained data are part of collaborative work for AUTOCC-ITS project for urban nodes interoperability tests [29]. The second driving path was meant to evaluate the LC system with the introduction of different detection confusion situations, where lanes-marking could be deficient, disconnected, or covered by on-road materials.

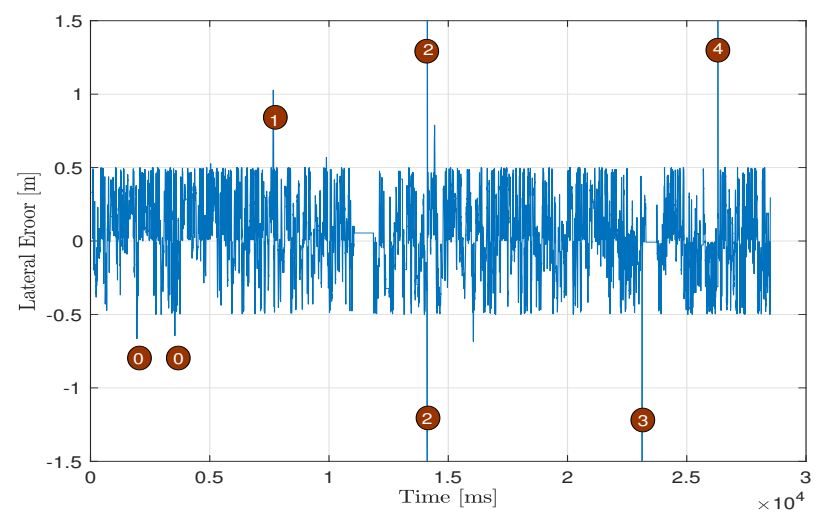

Fig. 9. Real-time lateral error sample data obtained during the $60 \mathrm{~km}$ tour on the M40 highway-Madrid. The data demonstrate the undetectable lane marks on the road, cases 2,3 , and 4 .

The results depicted in Fig. 9 record the spikes 0 and 1 , which represent a confusion when the camera detects an adjacent multi-lane scenario due to the relatively long intervehicle distance. Spikes 0 and 1 are still tolerable to achieve a reliable VLC communication over 10 meters of intervehicle distance (see Fig. 8). Other spikes $(2,3,4)$ can exceed the tolerable VLC lateral error (1 meter) which was the direct result of a temporary lane-marking deficiency along the driving path.

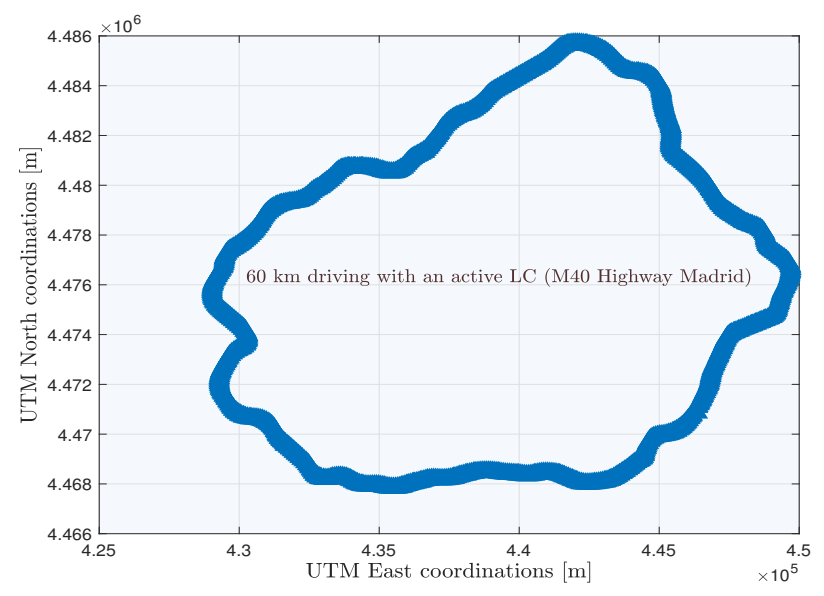

Fig. 10. $60 \mathrm{~km}$ of Lane centering (LC) driving route on the M40 highway Madrid. 


\section{CONCLUSION AND FUTURE WORK}

In this paper, we studied and evaluated the deployment of the LC application to enhance the (VLC) optical-based communication connectivity for a platoon of autonomous vehicles. The results obtained confirmed the reduction in the misalignment between platoon members and ensured the VLC link stability independently of any cooperation requirements when the road lanes are detectable. Other detection confusion or temporary lane-marking deficiency scenarios can be tolerated and might introduce a temporary VLC interruption. Compared to communication-based platoon and LRF -VLC systems, we believe that the presented approach of applying the correction locally by using LC makes the system more resilient and able to rapidly recover any communication interruptions. However, the presented work in this paper is an experiment-based investigation meant for concept validation. We conclude that the study needs to further extend the investigation cases by including different trajectory scenarios, weather conditions, and more accurate platoon controllers.

\section{ACKNOWLEDGEMENT}

This work has been supported by the European project AUTOC-ITS 2015-EU-TM-0243-S, where the obtained results are part of collaborative work between UPM-Madrid and INRIA-Paris during the urban nodes autonomous driving and interoperability validations tests.

\section{REFERENCES}

[1] S. Tsugawa, "Inter-vehicle communications and their applications to intelligent vehicles: an overview," in Intelligent Vehicle Symposium, 2002. IEEE, vol. 2, June 2002, pp. 564-569 vol.2.

[2] M. Abualhoul, "Visible Light and Radio Communication for Cooperative Autonomous Driving: applied to vehicle convoy," Theses, MINES ParisTech, Dec. 2016. [Online]. Available: https: //hal.inria.fr/tel-01447124

[3] D. Jiang and L. Delgrossi, "Ieee 802.11p: Towards an international standard for wireless access in vehicular environments," in Vehicular Technology Conference, 2008. VTC Spring 2008. IEEE, May 2008, pp. 2036-2040.

[4] J. J. Anaya, E. Talavera, D. Gimnez, N. Gmez, J. Felipe, and J. E. Naranjo, "Vulnerable road users detection using v2x communications," pp. 107-112, Sept 2015.

[5] Y. Wang, J. Hu, Y. Zhang, and C. Xu, "Reliability evaluation of ieee 802.11 p-based vehicle-to-vehicle communication in an urban expressway," Tsinghua Science and Technology, vol. 20, no. 4, pp. 417-428, August 2015.

[6] M. Y. Abualhoul, M. Marouf, O. Shagdar, and F. Nashashibi, "Platooning control using visible light communications: A feasibility study," in 16th International IEEE Conference on Intelligent Transportation Systems (ITSC 2013), Oct 2013, pp. 1535-1540.

[7] B. Béchadergue, L. Chassagne, and H. Guan, "Experimental comparison of pulse-amplitude and spatial modulations for vehicleto-vehicle visible light communication in platoon configurations," Opt. Express, vol. 25, no. 20, pp. 24790-24802, Oct 2017. [Online]. Available: http://www.opticsexpress.org/abstract.cfm?URI= oe-25-20-24790

[8] M. Abualhoul, M. Marouf, O. Shag, and F. Nashashibi, "Enhancing the field of view limitation of visible light communication-based platoon," in Wireless Vehicular Communications (WiVeC), 2014 IEEE 6th International Symposium on, Sept 2014, pp. 1-5.

[9] A. M. Cilean and M. Dimian, "Current challenges for visible light communications usage in vehicle applications: A survey," IEEE Communications Surveys Tutorials, vol. 19, no. 4, pp. 2681-2703, Fourthquarter 2017.
[10] Y. Wang, E. K. Teoh, and D. Shen, "Lane detection and tracking using b-snake," Image and Vision Computing, vol. 22, no. 4, pp. 269 - 280, 2004. [Online]. Available: http://www.sciencedirect.com/ science/article/pii/S0262885603002105

[11] S. Shladover, "Path at 20 - history and major milestones," in Intelligent Transportation Systems Conference, 2006. ITSC 'O6. IEEE, 2006, pp. 122-129.

[12] P. Daviet and M. Parent, "Longitudinal and lateral servoing of vehicles in a platoon," in Intelligent Vehicles Symposium, 1996., Proceedings of the 1996 IEEE, 1996, pp. 41-46.

[13] S. Hall, B. Chaib-draa, and J. Laumonier, "Car platoons simulated as a multiagent system," in In: Proc. 4th Workshop on Agent-Based Simulation, 2003, pp. 57-63.

[14] E. C. Tom Robinson, Eric Chan, "Operating platoons on public motorways: An introduction to the sartre platooning programme," in 17th World Congress on Intelligent Transport Systems (ITS) 2010, October 2010.

[15] S. Oncu, J. Ploeg, N. van de Wouw, and H. Nijmeijer, "Cooperative adaptive cruise control: Network-aware analysis of string stability," Intelligent Transportation Systems, IEEE Transactions on, vol. 15, no. 4, pp. 1527-1537, 2014.

[16] V. Milanés, S. E. Shladover, J. Spring, C. Nowakowski, H. Kawazoe, and M. Nakamura, "Cooperative adaptive cruise control in real traffic situations," Intelligent Transportation Systems, IEEE Transactions on, vol. 15, no. 1, pp. 296-305, 2014.

[17] A. Cailean, B. Cagneau, L. Chassagne, S. Topsu, Y. Alayli, and J.-M Blosseville, "Visible light communications: Application to cooperation between vehicles and road infrastructures," in 2012 IEEE Intelligent Vehicles Symposium (IV), 2012, pp. 1055-1059.

[18] M. Y. Abualhoul, O. Shagdar, and F. Nashashibi, "Visible light intervehicle communication for platooning of autonomous vehicles," in 2016 IEEE Intelligent Vehicles Symposium (IV), June 2016, pp. 508513.

[19] B. M. Masini, A. Bazzi, and A. Zanella, "Vehicular visible light networks with full duplex communications," in 2017 5th IEEE International Conference on Models and Technologies for Intelligent Transportation Systems (MT-ITS), June 2017, pp. 98-103.

[20] A. Demir and M. C. Macit, "Cooperative adaptive cruise control using visible light communication," in 2017 25th Signal Processing and Communications Applications Conference (SIU), May 2017, pp. 14.

[21] M. Y. Abualhoul, P. Merdrignac, O. Shagdar, and F. Nashashibi, "Study and evaluation of laser-based perception and light communication for a platoon of autonomous vehicles," in 2016 IEEE 19th International Conference on Intelligent Transportation Systems (ITSC), Nov 2016, pp. 1798-1804.

[22] Z. Guo, H. Liu, Y. Qian, and X. Wang, "A novel obstacle detection method based on distortion of laser pattern," pp. 1-6, July 2016.

[23] R. Behringer, S. Sundareswaran, B. Gregory, R. Elsley, B. Addison, W. Guthmiller, R. Daily, and D. Bevly, "The darpa grand challenge development of an autonomous vehicle," in IEEE Intelligent Vehicles Symposium, 2004, June 2004, pp. 226-231.

[24] M. R. Hafner, K. S. Zhao, A. Hsia, and Z. Rachlin, "Localization tools for benchmarking adas control systems," in 2016 IEEE International Conference on Systems, Man, and Cybernetics (SMC), Oct 2016, pp. $002665-002670$.

[25] J. W. Lee and B. Litkouhi, "A unified framework of the automated lane centering/changing control for motion smoothness adaptation," in 2012 15th International IEEE Conference on Intelligent Transportation Systems, Sept 2012, pp. 282-287.

[26] M. Abualhoul, M. Marouf, O. Shagdar, and F. Nashashibi, "Platooning control using visible light communications: A feasibility study," in Intelligent Transportation Systems - (ITSC), 2013 16th International IEEE Conference on, Oct 2013, pp. 1535-1540.

[27] J. E. Naranjo, C. Gonzalez, R. Garcia, and T. de Pedro, "Lane-change fuzzy control in autonomous vehicles for the overtaking maneuver," IEEE Transactions on Intelligent Transportation Systems, vol. 9, no. 3, pp. 438-450, Sept 2008.

[28] [Online]. Available: http://www.mobileye.com/en-uk/products/ mobileye-5-series/

[29] [Online]. Available: https://www.autocits.eu 